\title{
Are patients with newly diagnosed breast cancer getting appropriate DEXA scans? A District General Hospital experience
}

\author{
Huan Dong, Pete Dayananda, Shay-Anne Preece, Amtul Carmichael
}

Russells Hall Hospital, UK

\begin{abstract}
Breast cancer patients are often at high risk of fragility fractures partly due to adjuvant endocrine therapy such as aromatase inhibitors and chemotherapy. Baseline dual energy X-ray absorptiometry (DEXA) scanning is recommended as a standard of care in identifying patients who are at risk so they can be commenced on bone protective therapy.

NICE guideline 80 - "Early and locally advanced breast cancer"[1] states that patients with early invasive breast cancer should have a baseline DEXA scan to assess BMD before the commencement of aromatase inhibitor treatment; if patients have treatment-induced menopause or are starting ovarian ablation/suppression therapy. We have audited the performance of a DGH against these guidelines with a target of $100 \%$ concordance.

During a one year period (April 2012-April 2013), 100 patients with a new diagnosis of breast cancer were selected at random from the hospital coding database. 100 patients were chosen as this was a convenient sample size. We gathered information for these patients using electronic records, letters, and imaging. This showed a poor compliance of $38 \%$ against NICE guidelines. This in turn means that patients with low BMD at diagnosis of breast cancer are being under diagnosed and under treated, resulting in increased potential morbidity associated with fragility fractures. The interventions that resulted from this audit were: dissemination of these results to surgical and oncology departments, posters summarising the guidelines put up in breast clinics, and breast MDTs to discuss the need for DEXA scans for patients with breast cancer.

A re-audit was performed for patients diagnosed with early, invasive breast cancer in January 2014 where a compliance of $90 \%$ was achieved. This represents a huge improvement in compliance from the baseline measure of $38 \%$. In order to show that this improvement could be sustained, two further cycles were performed in February and March 2014, where the compliance was $92 \%$ and $100 \%$ respectively. Therefore the improvement in compliance was not only maintained but in fact the compliance increased even further during subsequent cycles.
\end{abstract}

Hence we have achieved a large improvement in the quality of assessment of bone quality in breast cancer patients. Moreover, we have demonstrated the importance of the dissemination of information and education within a multidisciplinary setting.

\section{Problem}

Patients with newly diagnosed with breast cancer are often started on treatments which can reduce their bone mineral density (BMD) and therefore increase the risk of fragility fracture. Early assessment of BMD and prophylactic bone protective therapy is key to limiting the risk of fragility fractures and its associated morbidity.

There is clear national guidance contained within NICE guideline 80 - Early and locally advanced breast cancer[1] which states that:

- Patients with early invasive breast cancer should have a baseline dual energy X-ray absorptiometry (DEXA) scan to assess bone mineral density if they:

- are starting adjuvant aromatase inhibitor treatment,

- have treatment-induced menopause,

- are starting ovarian ablation/suppression therapy.
- Do not offer a DEXA scan to patients with early invasive breast cancer who are receiving tamoxifen alone, regardless of pretreatment menopausal status.

Therefore our aim was to audit our practice at Russells Hall Hospital, Dudley against these guidelines and then to make changes resulting in a significant quality improvement.

\section{Background}

Osteoporosis is defined as a skeletal disorder characterised by compromised bone strength predisposing to an increased risk of fracture[1], and in terms of BMD is defined by the WHO as a Tscore of less than-2.5 SD. Osteoporosis is known to be particularly prevalent in postmenopausal women due to reduced circulating oestrogen levels. Oestrogen is known to inhibit osteoclast activity[3] and therefore any reduction in its levels will cause an increase in bone loss.

Patients diagnosed with breast cancer are often started on 
treatments which reduced their oestrogen levels and therefore reduce their BMD. This includes the use of aromatase inhibitors, ovarian suppression or ablation therapy, and chemotherapy induced premature menopause. Aromatase inhibitors are increasingly used in postmenopausal patients who are ER-positive, as they have been shown to reduce the risk of recurrence even more than tamoxifen, which is the previous standard of care. They work by inhibiting the aromatase enzyme and therefore reducing the conversion of androgens to oestrogen, thereby suppressing oestrogen levels to almost undetectable levels. However, in postmenopausal women aromatase inhibitors have been shown to cause bone loss at sites rich in trabecular bone at an average rate of $1-3 \%$ per year[2] leading to increased incidence of fracture compared to tamoxifen alone.

In contrast, tamoxifen works as an oestrogen agonist in postmenopausal women and so actually increases BMD, and this may lead to a reduction in risk of fractures. In pre-menopausal women, tamoxifen works as an oestrogen antagonist, causing a reduction in BMD, but the loss is only about $1-2 \%$ and the effect does not persist through five years of tamoxifen therapy[2]. Hence, routine DEXA scanning in not recommended in patients on tamoxifen alone.

In premenopausal women, premature ovarian suppression will also cause a reduction in oestrogen levels whether that is due to gonadotrophin-releasing hormone $(\mathrm{GnRH})$ agonists, cytotoxic chemotherapy, or surgical ablation. Therefore, as expected, ovarian suppression/ablation and chemotherapy induced premature menopause will also reduce BMD and baseline DEXA scanning is recommended for these patients.

Patients found to have low BMD (i.e. osteoporotic/osteopaenic) on DEXA scanning should then be started on appropriate bone protection therapy such as bisphosphonates and calcium/Vit $D$ in order to prevent further bone loss induced by breast cancer treatment. This will in turn reduce the morbidity and mortality associated with fragility fractures such as hip and spinal fractures.

\section{Baseline measurement}

A retrospective audit of case notes of patients with newly diagnosed breast cancer was performed. 100 patients with a new diagnosis of breast cancer between April 2012 and April 2013 were randomly selected from the hospital coding database. Electronic letters were used to identify if these patients had early and locally advanced breast cancer and if so whether they met criteria for DEXA scanning as per NICE guidelines. Radiology records were searched for each patient to see if DEXA scans were performed and results of scans recorded. Records were reviewed to ensure a minimum follow-up of 1 year.

Out of 100 patients with new diagnosis of breast cancer, 66 had early and locally invasive breast cancer. The others included 25 patients that did not meet criteria for locally invasive breast cancer (e.g. metastatic disease) and 9 who had inadequate data. Of these 66 women, 42 had indications for DEXA scanning: all were for aromatase inhibitors. Only $38 \%$ (16/42) of patients who met the NICE guidelines for DEXA imaging received DEXA scans. Of those who were scanned, over half had some evidence of bone loss: $50 \%$ $(8 / 16)$ were osteopaenic and $6 \%(1 / 16)$ were osteoporotic.

This showed that there was a very poor compliance with NICE guidelines which means that a large proportion of patients would not receive bone protection therapy potentially leading to an increase in preventable fragility fractures.

See supplementary file: ds6014.pptx - "PDSA cycle 1 results"

\section{Design}

The findings of the baseline measurement were presented and discussed at the local general surgical audit meeting (which included breast surgeons). It was felt that the most important aim was to disseminate these guidelines and educate clinicians to follow them. Whilst it was important for breast surgeons to be aware of these guidelines, we felt that it was even more important for the whole multidisciplinary team to be aware of and follow these guidelines as it requires input from many healthcare professionals to start patients on adjuvant therapy such as aromatase inhibitors and follow patients up during chemotherapy. To this end the results of this audit were disseminated to the MDT. Moreover, posters were constructed, clearly highlighting the guidelines and placed in prominent clinical areas such as breast clinics and MDTs.

\section{Strategy}

PDSA Cycle 1: 100 case records were reviewed as a baseline to assess how compliant we were with NICE guidelines regarding DEXA scanning in breast cancer patients. This revealed a low compliance of only $38 \%$ and so there was much room for improvement. We felt that a poor knowledge of these guidelines were the main problem and therefore widely disseminated the guidelines to both surgical and oncology departments using a mixture of presentations and posters.

PDSA Cycle 2: Case records were reviewed for patients diagnosed with early breast cancer during January 2014 to look for an improvement in compliance. Out of 22 patients diagnosed with early breast cancer, 10 had indications for DEXA scanning. Out of those 10 patients, 9 received DEXA scans which gives a compliance of $90 \%$. Results were fed back to members of the breast MDT to encourage sustained improvement.

PDSA Cycle 3: Case records were reviewed again for patients diagnosed with early breast cancer during February 2014 to look for sustained improvement. Out of 22 patients diagnosed with early breast cancer, 13 had indications for DEXA scanning. Out of those 13 patients, 12 received DEXA scans which gives a compliance of $92 \%$.

PDSA Cycle 4: Case records were reviewed again for patients diagnosed with early breast cancer during March 2014 to look for sustained improvement. Out of 17 patients diagnosed with early breast cancer, 11 had indications for DEXA scanning. Out of those 11 patients, all 11 received DEXA scans which gives a compliance 
of $100 \%$. This clearly shows that our interventions have not only improved compliance but that this improvement has been sustained.

\section{Results}

A re-audit was performed for patients diagnosed with early, invasive breast cancer in January 2014 after the implementations had been bedded in. There were 22 patients diagnosed with early invasive breast cancer in January 2014, of which 10 needed DEXA scanning according to NICE guidelines. Out of these 10 patients only 1 patient did not receive a DEXA scan and therefore the compliance was $90 \%$. This represents a huge improvement in compliance from the baseline measure of $38 \%$. The results were fed back to the breast cancer MDT in order to encourage everyone to maintain the improvement.

In order to show that this improvement could be sustained, 2 further cycles were performed for patients diagnosed with early invasive breast cancer in February and March 2014. In February and March, the compliance was $92 \%$ and $100 \%$ respectively. This shows that not only was the improvement maintained but in fact the compliance increased even further during subsequent cycles.

See supplementary file: ds6163.pptx - "Run chart of data"

\section{Lessons and limitations}

This audit has demonstrated the compliance with the NICE guidelines can be enhanced by clear communication and dissemination of information in a multidisciplinary setting.

However there were limitations associated with this audit. The first issue is that there were a significant number $(16 \%)$ of patients whose records were incomplete or did not contain enough information to accurately assess whether they met the guidelines or not. Hence they were excluded from the results - as shown in the flow diagrams attached.

In addition, the relatively frequent turnover of patients within the surgical and oncology departments means that there could potentially be problems with continuity and maintenance of the current high level of compliance. This is not so much of a problem with consultants but can be a problem with trainees on rotational placements. Perhaps one solution would be to include these guidelines in the induction booklets given to trainees at the start of their placements. Further cycles will need to be performed in the future to ensure that high compliance is maintained.

\section{Conclusion}

Our interventions have clearly achieved a significant improvement in compliance with NICE guidelines on DEXA scanning in breast cancer patients from $36 \%$ in the first cycle to $100 \%$ in the fourth cycle. This demonstrates the importance of good dissemination of information and education in a multidisciplinary setting. With a significant number of patients found to be osteoporotic/osteopaenic on DEXA scanning, we would like to think that appropriate bone prevention therapy will have been started and that we have reduced the number of potential fragility fractures in this cohort.

Future quality improvement work could aim to see if these patients identified to have low BMD were actually commenced on the appropriate bone protective treatment, as outlined in the same NICE guideline (80)

\section{References}

1 National Institute for Health and Clinical Excellence (2009) Early and locally advanced breast cancer: Diagnosis and treatment (CG80)

2 Reid DM, Doughty J, Eastell R et al. Guidance for the management of breast cancer treatment-induced bone loss: A consensus position statement from a UK Expert Group. Cancer Treat Rev 2008; 34 (Suppl 1): S3-S18

3 Krassas GE, Papadopoulou Ph. Oestrogen action on bone cells. J Musculoskel Neuron Interact 2001; 2(2): 143-151

\section{Declaration of interests}

Nothing to declare

\section{Acknowledgements}

Dr Andrew Whallett

\section{Ethical approval}

This work was deemed an improvement study and not a study on human subjects, and therefore as per local policy, ethical approval was not required. 